\title{
Sex Differences in Salivary Cortisol Responses to Sex-Linked Stressors: A Test of the Tend-and-Befriend Model
}

\author{
Jennifer Byrd-Craven • Brandon J. Auer • \\ Shelia M. Kennison
}

Received: 10 July 2014 / Accepted: 20 October 2014 /

Published online: 25 October 2014

(C) Springer International Publishing 2014

\begin{abstract}
This study examined sex differences in salivary cortisol responses to experimental stressors and subsequent desire to affiliate in the context of mortality cues following the Tend-and-Befriend model. Participants were randomly assigned to a mortality salience prime or a control condition. They were then assigned to view a video of crying infants or a video designed to elicit out-group threat. As predicted, females showed more cortisol reactivity to the video of crying infants and males showed more cortisol reactivity to the video of hate speech in the no prime condition. Males receiving a mortality salience prime, surprisingly, showed a weaker cortisol response to the out-group threat video compared to males who received no prime. Results provide further support of using the Tend-and-Befriend model to predict differences in stress response system activity between men and women, as well as modifications to that theory that incorporate male responses to out-group threats.
\end{abstract}

Keywords Sex differences · Tend-and-befriend $\cdot$ Cortisol $\cdot$ Mortality salience

\section{Introduction}

Identifying and predicting sex differences in biobehavioral responses to environmental stressors and the experience of psychological stress has been of significant interest in psychobiological research. Taylor et al. (2000) outlined an evolutionary theoretical framework for predicting sex differences in biobehavioral responses to stress. Briefly, the model notes key differences between male and female response patterns to stressors - males are expected to closely follow the classic fight-or-flight response pattern, indicative of sympathetic nervous system activity, while females are expected, in some contexts, to follow a behavioral pattern that provides care and protection for offspring when present (tending component) as well as seek affiliation and social

\footnotetext{
J. Byrd-Craven $(\bowtie) \cdot$ B. J. Auer $\cdot$ S. M. Kennison

Department of Psychology, Oklahoma State University, Stillwater, OK, USA

e-mail: jennifer.byrd.craven@okstate.edu
} 
support with a group that is ideally composed of the same sex (befriending component). This tend-and-befriend model of stress reactivity maintains that the fight-or-flight response does not always make sense as an initial response to challenges for females; rather, the dominant response is to build or draw on social networks (befriending) as means of reducing stress, and that the hypothalamic-pituitary-adrenal (HPA) axis and oxytocin systems are preferentially activated during stress. These networks are thought to underlie the reduced focus on dominance hierarchies and related status-seeking behavior in female social structure (Campbell 1999; Geary 2010).

There is considerable empirical support for using the tend-and-befriend model to predict sex differences in biobehavioral responses to environmental stressors (e.g., Stroud et al. 2002, 2009). Related to 'befriending', women appear to use affiliation as a mechanism to reduce stress and appear to be particularly sensitive to gaps in social support networks (Taylor 2006). This is consistent with findings that show that social support is particularly important for females throughout the lifespan, especially during the reproductive years. The existence and availability of same-sex friendships serves as a core source of social support for females during adolescence and through at least young adulthood (Gabrill and Kerns 2000; Rubin, Bukowski, and Parker 1998). The support processes that occur within these relationship interactions have been shown to be adaptive and protective in nature (Uchino, Uno, and Holt-Lunstad 1999), and are associated with a lower incidence of stress-related psychopathology (Taylor 2006).

Part of the 'tending' response in women may include sensitivity to cues involving infant distress. As the tending predisposition is likely to be regulated, in part, by activity of the nervous system, females would be expected to demonstrate physiological responsiveness to cues of infant distress, such as crying. Such responsiveness would be expected to facilitate tending behaviors, particularly in cooperatively breeding species such as humans (Hrdy 2007). As a product of the HPA axis, cortisol is an ideal candidate for indexing sensitivity to cues of infant distress due to its positive association with maternal behavior and responsiveness following parturition (Fleming et al. 1997a, b). Further suggesting a potential role for cortisol in facilitating tending behavior, first-time adult mothers at 2-3 days postpartum with higher baseline measurements of salivary cortisol engaged in more affectionate contact behaviors as well as more caretaking activities (Fleming et al. 1997b). Moreover, when exposed to audio recordings of infants crying, postpartum mothers with higher cortisol levels and heart rates experienced greater sympathy, demonstrating that activity of the HPA system facilitates responding to infant cues (Stallings, Fleming, Corter, Worthman, and Steiner 2001). Evidence of cortisol sensitivity in the context of infant cues is not limited to mothers, however; teenaged and adult females who are not mothers show greater cortisol responses to an audio recording of infants crying compared to an audio recording of 'neutral' verbal passages being read (Giardino, Gonzalez, Steiner, and Fleming 2008).

Notably, Geary and Flinn (2002) suggested modifications to the Tend-and-Befriend model to extend to males as well, highlighting the likely importance of male philopatry - the tendency for males to stay in their birth group and for females to emigrate upon marriage - in human evolution. Following this modification, both men and women form coalitions that facilitate stress reduction among members of the group, as opposed to the tend-and-befriend model's primary emphasis on stress reduction in female groups. Men have historically operated within kin-based coalitions, where cooperation was likely used to maintain coalitions. Competition within male coalitions potentially increased status and access to reproductive resources of individual 
members within the group. Once established, dominance hierarchies facilitate cooperation needed for coalitional actions against competing coalitions. The more cooperation - and cohesion or identification with the coalition - the better that coalition functions as a unit against other coalitions. Importantly, the disposition to form coalitions is primarily expressed under conditions of social threat (Geary 2010; Savin-Williams 1979), and the threat of an out-group represents a source of social stress in males that would be expected to trigger hormonal activity as part of a coordinated stress system response. Victories against outside members of a group, for example, are associated with elevations in testosterone in human (Bernhardt et al. 1998; Gonzalez-Bono et al. 1999; 2000; Kivlighan et al. 2005; Oxford et al. 2010; Wagner et al. 2002) and animal studies (Fuxjager et al. 2009; Gleason et al. 2009). Further, higher levels of pre-competition testosterone and cortisol are associated with reduced strength of coalitionary ties (Flinn, Ponzi, and Muehlenbein 2012). Presumably, higher levels of testosterone and cortisol may interfere with in-group formation (Oxford, Ponzi, \& Geary 2010). The relationship between stress system response and coalitionary ties can vary with level of expertise and previous experience, and investment in the outcome (Kivlighan et al. 2005). There is additional evidence that cortisol may be elevated in the context of social out-group threats. In another recent study, cortisol responses predicted subtle and blatant prejudice in people anticipating having an interaction with a member of an out-group who was dissimilar to the self (Bijleveld, Scheepers, and Ellemers 2012).

While cues of infant distress and out-group threat potentially contribute to physiological reactivity and affiliative responses in females and males, respectively, reminding individuals of their mortality presents another source of acute stress that may enhance biobehavioral responses to sex-specific stressors. Terror management theory (TMT) is a broad theory highlighting the role that knowledge of inevitable mortality plays in a diverse range of human behaviors (Solomon, Greenberg, and Pyszczynski 1991). TMT posits that humans possess an instinctive, biological drive for self-preservation but also have an understanding of the inevitability of death, which can contribute to existential anxiety (Greenberg, Pyszczynski, and Solomon 1986; Solomon, Greenberg, and Pyszczynski 1991). The mortality salience (MS) hypothesis of TMT suggests that reminding people of their mortality (MS induction) should increase their need for the structure and meaning provided by their personal worldview, need for self-esteem, and desire for close, personal relationships. Experimental evidence suggests that induction of MS promotes worldview defense in the form of greater emphasis placed on personally and culturally valued factors, and extrinsic goals such as physical attractiveness, materialism, and wealth (Arndt et al. 2004; Kasser and Sheldon 2000; Routledge, Arndt, and Goldenberg 2004). MS has also been linked to increases in positive evaluations of in-groups and negative evaluations of out-groups (McGregor et al. 1998). Of particular relevance to the present study, MS has been associated with increased affiliation as indexed by sitting with a group rather than sitting alone (Wisman and Koole 2003). Further, some sex differences have been documented following MS induction. Notably, MS primes have been found to increase accessibility of nationalistic constructs for men, but not women. MS induction has also been associated with increased romantic accessibility in women, but not men (Arndt, Greenberg, and Cook 2002).

Following the tend-and-befriend model and its modification by Geary and Flinn (2002), both males and females would be expected to desire and seek affiliation following the experience of stress. Sex differences may vary, however, depending on the type of stressors that initiate biobehavioral responses in males and females. 
Specifically, women are expected to be more physiologically and psychologically sensitive to environmental cues related to caretaking, such as the sound of infants crying, while males are expected be more sensitive to cues related to a perceived outgroup threat, for example, aggressive or inflammatory speech directed at their coalition. The mortality salience (MS) hypothesis of TMT suggests that reminding people of their mortality (MS induction) should increase the desire to affiliate and seek social contact with others. Further, MS induction is also expected to enhance physiological stress system reactivity to sex-specific stressors in males (i.e., out-group threat) and females (i.e., cues of infant distress). It is important to note that we are not suggesting that outgroup threat will not elicit stress system activity in females, nor should cues of infant distress fail to elicit a response in males - the sex differences in physiological reactivity proposed here are relative, and not exclusive.

To date, there have been limited attempts to directly assess Geary and Flinn's (2002) suggested modifications to the Tend-and-Befriend theory. This study seeks to address three questions that will significantly contribute to the available literature on stress system activity and affiliative responses in the context of sex-specific stressors and mortality salience. Specifically, the following questions were addressed: (1) Are changes in cortisol associated with the hypothesized sex-specific stressors of out-group threat for males and infant distress in females? (2) Do the hypothesized sex-specific stressors increase the desire to affiliate in men and women? The Tend-and-Befriend model and its associated modifications suggest that both men and women should desire affiliation when exposed to salient environmental stressors such as the sound of infant distress or out-group threat. (3) Are changes in cortisol associated with mortality salience induction? MS induction may enhance stress responses to sex-specific stressors. As such, increased cortisol responses following MS induction are predicted in men exposed to out-group stressors (i.e., inflammatory speech) and in females exposed to cues of infant distress (i.e., crying).

\section{Materials and Methods}

Participants

One hundred forty two healthy undergraduate psychology students (73 women, 69 men) were recruited for the study as part of course credit. Participants were aged 1827 years, and were screened for factors known to potentially influence cortisol levels (e.g., use of caffeine, hormonal contraceptives, and medication, illness, etc.). Women and men were recruited in same sex groups of 3-4 individuals and were randomly assigned to a mortality salience (MS) or control (dental pain) condition. After receiving a complete description of the study, written informed consent was obtained. All procedures were reviewed and approved by the Institutional Review Board (IRB).

\section{Measures}

\section{Questionnaires}

Participants first completed a battery of questionnaires as a part of a larger study. Time spent to complete the questionnaires on demographics, personality and thinking styles 
also served to habituate participants to the novel laboratory setting. Following exposure to laboratory stressors, participants were asked to rate on a 1-7 Likert-type scale their desire to affiliate with their fellow participants, their desire to affiliate with those with whom they had close relationships, and their level of overall distress.

\section{Cortisol}

Saliva was obtained by having participants hold a $1 \times 4 \mathrm{~cm}$ absorbent swab in their mouth for 1-2 min. Saliva-saturated swabs were stored at $-20{ }^{\circ} \mathrm{C}$ until assayed at Oklahoma State University. On the day of testing, saliva samples were centrifuged at 3,000 rpm for $15 \mathrm{~min}$ to remove mucins. Following Granger and colleagues (2007), samples were assayed cortisol (enzyme immunoassay) using commercially available reagents (Salimetrics, State College, PA, USA) without modification to the manufacturer's recommended protocols. Cortisol levels are reported in micrograms per deciliter (ug/dL). These assays had average intra- and inter-assay coefficients of variation less than 5 and $15 \%$, respectively.

\section{Procedure}

Male and female participants first completed a battery of questionnaires (approximately 10 $15 \mathrm{~min}$ ), followed by the collection of a baseline saliva sample. See Fig. 1 for sample collection times. Participants were then randomly assigned to receive a mortality salience prime (experimental condition) or a dental pain prime (control condition). Participants in the morality salience prime condition were told to "Please briefly describe the emotions that the thought of your own death arouses in you. Please describe, as specifically as you can, what you think will happen to you as you physically die and once you are physically dead." Subjects in the control condition were told to "Please briefly describe the thought of experiencing dental pain arouses in you. Please describe, as specifically as you can, what you think will happen as you experience dental pain and once you are experiencing dental pain." Completion of this condition took approximately 5 min to complete. Next, participants were presented with either male typical 'out-group threat' stressor consisting of video footage of the Westboro Baptist Church military funeral protests, which featured statements such as "God hates America," "Dead soldiers are God's wrath for your sins," and "your family is cursed", or a female typical stressor of crying infants. Each recording lasted 5 min and all subjects were seated equal distances from the monitor. A second saliva sample was collected directly following completion of the audio/video clips. Participants were then asked to fill out a series of questionnaires regarding their emotional state, desire to affiliate with those closest to them (e.g., family, close friends, romantic partner), and their overall perception of the video clip (on a 1-5 scale). They were then asked to fill out a filler questionnaire on thinking styles, and once finished were asked to sit quietly and refrain from interacting with the other group members. Once $20 \mathrm{~min}$ had elapsed from the video stressor,

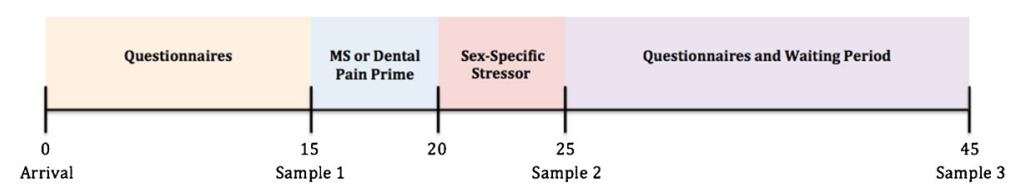

Fig. 1 Timeline of experiment procedure and saliva sampling 
a final saliva sample was collected to capture cortisol at its associated peak (Gordis et al. 2006) (see Fig. 1).

\section{Data Analysis}

To determine the reaction to the video stressors on cortisol reactivity, a three-way ANOVA was computed using Mortality Salience group (MS or dental prime), video group (babies crying or out-group threat), and sex (male or female) as the betweenparticipant independent variables, and cortisol change scores as the dependent variable. Table 1 shows the number of participants per group. Though the sample sizes among the groups were unequal (due to random assignment to groups), in order for unequal sample sizes to affect statistical conclusions, they must be different at a proportion of 4 to 1 (Tabachnick and Fidell 2007). Because departure from normality also affects statistical conclusions, we also examined outliers. Two samples were identified as outliers (more than three standard deviations from the mean), one male in the control/out-group threat condition (3 standard deviations below the mean) and one female in the MS/out-group threat condition ( 3 standard deviations above the mean). The analyses described below were run first with the outliers included and then with them excluded. Excluding the outliers did not change the pattern of results, and thus results reported below include the outliers. The cortisol change score was computed to determine the change from pre-task to post-task by subtracting the post-task from pre-task cortisol scores. This method of estimating reactivity via change scores was chosen over area under the curve (AUC) due to the relatively limited number of samples collected. In studies with less than four data collection points, AUC is unlikely to yield information over that of change scores (Pruessner, Kirschbaum, Meinlschmid, and Hellhammer 2003).

\section{Results}

\section{Cortisol}

There were no significant results in cortisol reactivity from Time 1 to Time $2(p=.18)$. However, this was not unanticipated, as cortisol typically takes 15-20 min post-stressor to peak (Gordis et al. 2006).

As predicted, there was a MS Group x video group $\mathrm{x}$ Sex interaction for cortisol reactivity (Time 1 to Time 3), $F(1,134)=4.09, p=.001$ (See Fig. 2). This interaction was then broken down into an analysis of sex X video group at each level of MS Group (one-tailed). These comparisons were carried out as one-tailed tests because of the

Table 1 Number of participants per condition

\begin{tabular}{llllll}
\hline & \multicolumn{2}{c}{ Control prime } & & & \multicolumn{2}{l}{ Mortality salience } & \\
\cline { 2 - 3 } \cline { 5 - 6 } & Babies crying & Out-group threat & & Babies crying & Out-group threat \\
\hline Female & 15 & 18 & 16 & 24 \\
Male & 14 & 11 & 21 & 23 \\
\hline
\end{tabular}




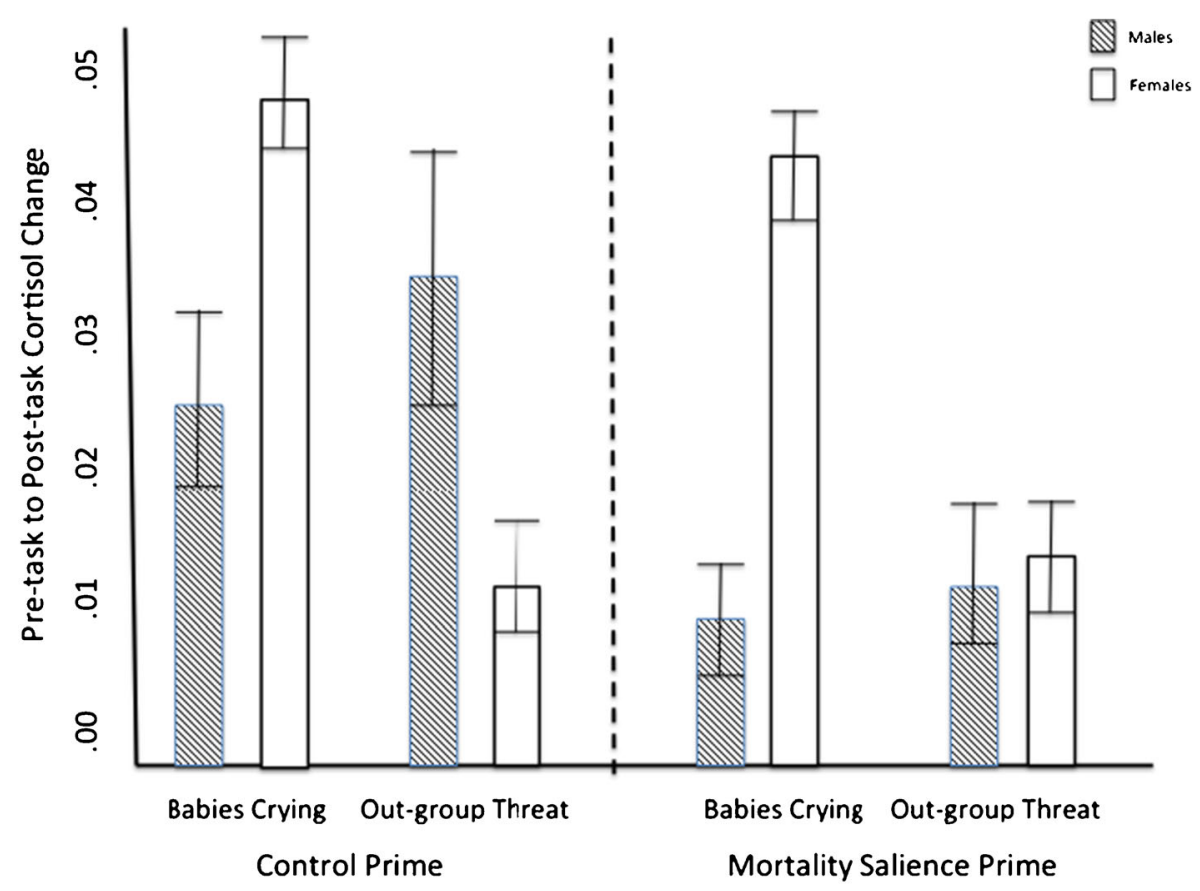

Fig. 2 Mean differences between males and females in cortisol change ( $\mu \mathrm{g} / \mathrm{dl})$ across conditions. Bars denote standard errors

directional nature of the hypothesis; specifically, participants in MS condition were predicted to experience increased out-group bias than participants in the control condition. Observing no difference between the MS and control condition or greater out-group bias in the control condition than in MS condition would lead to the acceptance of the null hypothesis (Nolan and Heinzen 2008, p. 361). In the MS control condition (dental prime), there were no significant sex differences in the babies crying condition, $t(28)=.729, p=.57$, but the out-group threat condition did show sex differences, $t(28)=1.92, p=.01$. In the MS condition (MS prime), the opposite pattern was found. There were significant sex differences in the babies crying condition, $t$ (36) $=2.93, p=.009$, but not in the out-group threat condition, $t(46)=0.67, p=.50$. Figure 2 displays the mean salivary cortisol change scores (pre-task to post-task) in men and women. These post-hoc analysis showed that the out-group threat video resulted in HPA reactivity for men, but that the mortality salience prime eliminated that effect. Surprisingly, males in the MS prime condition actually showed a suppressed cortisol response to the video of inflammatory speech compared with males in the MS control condition.

\section{Affiliation and Distress Data}

To determine whether there were group or sex differences in affiliation and distress after the video stressors, two separate three-way ANOVAs were computed using Mortality Salience group (MS or dental prime), video group (babies crying or outgroup threat), and sex (male or female) as the between-participant independent 
variables, and ratings of desire to affiliate and distress as the dependent variable. There were no group, $F(1,134)=.90, p=.46$, or sex differences, $F(1,134)=1.51, p=.21$, regarding the desire to affiliate with group members after the video stressors, nor was the interaction significant, $F(1,156)=1.63, p=.12$. Similarly, there were no group, $F(1$, $134)=.07, p=.85$, or sex differences, $F(1,134)=.51, p=.44$, regarding desire to affiliate with close relationships after the video stressors, nor was the interaction significant, $F(1,134)=.71, p=.40$. There were also no group, $F(1,134)=1.48, p=.26$, or sex differences, $F(1,134)=2.25, p=.06$, regarding overall ratings of distress after watching the video, though the sex difference was marginally significant, with females rating their distress higher than males. The interaction between group and sex was not significant, $F(1,134)=.22, p=.83$.

Correlations between desire to affiliate with group members, close relationships, and distress were run with cortisol at all three time points. There were no significant correlations between cortisol and any of the affiliation measures $(r \mathrm{~s}<.02)$, and this was also true when the correlations were divided by sex $(r \mathrm{~s}<.08)$ and experimental condition $(r \mathrm{~s}<$. There were significant correlations between distress ratings after the video and cortisol at Time 2, $r=.18, p=.03$, and Time $3, r=.17, p=.04$, but not at Time $1, r=.06, p=.49$, as expected. There were no differences in this pattern across experimental condition or sex $(r \mathrm{~s}<.13)$.

\section{Discussion}

This study addressed three important questions that have received sparse treatment in the literature to date. These questions concern (1) how changes in cortisol are associated with hypothesized sex-specific stressors of out-group threat for males and infant distress in females, (2) how exposure to sex-specific stressors are related to the desire of affiliation, and (3) how changes in cortisol are associated with mortality salience induction.

With respect to the first question, the tend-and-befriend model and its suggested modifications raise the possibility that males and females are sensitive to different psychosocial stimuli. Females were expected to show an enhanced sensitivity to cues of infant distress, eliciting a greater cortisol response compared to males. In contrast, males were expected to have an enhanced sensitivity to cues of out-group threat, as indicated by greater cortisol response to a video of inflammatory speech, relative to females. Results confirmed these predictions, where women displayed greater cortisol responses the stressor that elicited 'tending' responses (babies crying), and men displayed greater cortisol responses to threats to the in-group by a clear out-group (protests of military funerals). This is consistent with literature on sex differences in stress responses related to psychosocial stressors in a laboratory setting (Stroud et al. 2002, 2009). Perhaps more importantly, these results also lend empirical support to Geary and Flinn's (2002) predictions that males show greater physiological sensitivity to out-group threat, and females show greater physiological sensitivity to cues related to 'tending.' Thus, males do, in effect, show a response consistent with 'befriending' if befriending is conceived to encompass coalitional activity. The cortisol response observed in males within the current study may reflect sensitivity to out-group threat; heightened physiological sensitivity to cues of out-group threat may assist males to 
mount an effective behavioral response. This is consistent with other literature on this topic using cortisol and other biomarkers (DeSoto et al. 2010; Flinn et al. 2012; Oxford et al. 2010).

With respect to the second question, based on Geary and Flinn's (2002) modification of the tend-and-befriend model, it was predicted that when exposed to sex-specific stressors such as infant distress for females and out-group threat for males, both sexes would have an increased desire for affiliation. Our results showed that all participants reported a desire to affiliate with experimental group members as well as with close relationships, and that this did not differ by sex or by experimental group. The lack of the sex difference in this reported measure is noteworthy in light of the differences in physiological reactivity to the stressors. However, it is possible that the tasks lacked realism, thereby not eliciting responses similar to those outside the laboratory.

With respect to the third question, some data suggest that MS induction may enhance stress responses to sex-specific stressors. Increased cortisol responses were predicted in men exposed to an out-group stressor (inflammatory speech/funeral protest) following MS induction and in females exposed to cues of infant distress (i.e., crying). The results of the morality salience prime were surprising, and it is possible that the MS priming procedure was simply not that effective. We predicted that the experience of considering one's own death would intensify the reported and physiological response to these stressors. We found, however, that this was not the case. In fact, men showed a suppressed cortisol response to the out-group threat video when they had received a mortality salience prime prior to viewing the video. Some research has shown that young men measuring high in aggressive behavior (e.g., conduct disorder) show a dampened stress response to laboratory stressors (Gordis et al. 2006). Men have also been shown to have lower pre-competition cortisol levels when coalitionary ties are high (Flinn et al. 2012). Thus, our results may indicate that mortality salience serves to increase coalitionary ties, and possibly prepare for an aggressive response against an out-group. Further, mortality salience has been shown to increase weapon bias for members of an out-group, lending further support to the idea that it may be related to aggressive or defensive responses, though this finding was true for both sexes (Bradley and Kennison 2012). The weapon bias task, however, requires quick reaction times, which differs from the requirements of the task tested here in that this task allows for more time to consider a response.

A possible explanation for reduced cortisol reactivity in the face of out-group threat and mortality salience includes decreased adrenal sensitivity produced through other hormones implicated in biohehavioral responses to social stressors. The blunted cortisol response was an effect limited to men in this study, suggesting that androgens may play some role in the unexpected finding. Substantial evidence suggests that reproductive steroids modulate the response to stress. Supportive data include observations of sex differences in hypothalamic-pituitary-adrenal (HPA) axis activity/responsivity (McCormick et al. 2002; Roelfsema et al. 1993; Greenspan et al. 1993) and demonstration of acute regulatory effects of gonadal steroids on the HPA axis in animal castration and replacement studies (Burgess and Handa 1992; Critchlow et al. 1963; Bingaman et al. 1994). Free testosterone in particular would serve as an excellent candidate biomarker for elucidating the unexpected decreases in cortisol observed in young men exposed to the MS prime and out-group threat. Levels of free testosterone may increase in face of threat or challenge, particularly in response to social stressors 
that threatened status within a social hierarchy. Data is also available suggesting that the effect of testosterone in young men is to inhibit the cortisol response to stressors such as corticotrophin-releasing-hormone $(\mathrm{CRH})$ stimulation/challenge. For example, compared with the leuprolide-induced hypogonadal state, testosterone replacement produced a blunted cortisol response, decreased Area Under the Curve (AUC), as well as decreased cortisol MAX and delta MAX (Rubinow and Schmidt 1996). These findings are consistent with studies in rodents revealing a suppressive rather than enhancing effect of testosterone on stimulated HPA axis activity (Handa et al. 1994; Bingaman et al. 1994). Further, direct associations between testosterone, rates of aggression, and dominance rank have been identified in several species, including nonhuman primates (Bercovitch and Strum 1993).

Mortality salience may enhance coalitionary ties and prepare males for aggressive responses to an out-group, perhaps in part, due to modulation of cortisol and other hormones implicated in reactivity to social stressors, such as testosterone. However, the connection between cortisol and other hormones accounting for our unexpected finding is only speculative and warrants attention in future studies. Furthermore, there is a considerable amount of within-sex variability in physiological responses to stressors. The sex differences found here are relative, not exclusive, and future research should attend to within-sex variability as well.

This study is limited in its use of a single biomarker (cortisol). This is particularly of concern in relation to the surprising results related to men in the mortality salience/outgroup threat condition. Cortisol is one of many in a sequence of neurohormonal mechanisms that regulate responses to social threats and challenges (Byrd-Craven, Granger, and Auer 2011; Flinn 2006; Kivlighan, Granger, and Booth 2005). The use of additional biomarkers such as testosterone and perhaps the autonomic nervous system marker, salivary alpha-amylase, would provide the necessary evidence as to whether this response is, indeed, a preparation for aggressive response toward a threatening out-group. Further, ovulation status was not controlled for in women. Prior research has shown that the timing of the menstrual cycle influences how women are affected by MS when making mate judgments (Vaughn, Bradley, Byrd-Craven, and Kennison 2010), but no known prior research has investigated the impact of ovulation on MS affects on responses toward crying infants. Examining the impact of menstrual cycle changes on the effectiveness of MS primes in this paradigm would be an important future direction.

To our knowledge, this is the first study to provide a direct test of the physiological mechanisms that underlie Geary and Flinn's (2002) suggested modifications to Taylor and colleagues tend-and-befriend hypothesis. By incorporating a clear out-group threat, we showed that men are particularly sensitive to this type of social challenge, as indexed by cortisol reactivity. Perhaps more perplexing, we showed that this response differs based on whether they were first primed with their own mortality. Thus, in males at least, mortality cues may serve to influence physiology and associated behavior related to out-group challenges, though the exact mechanisms and direction is yet unclear. Future studies should incorporate a wide variety of biomarkers in order to better determine how these systems are interacting to influence cognition and behavior, with testosterone as a promising candidate for examination. It is notable that the mortality salience prime did not significantly impact women's responses to the 'tending' stressor. It appears that the HPA response is more sensitive to changes in 
mortality status as it is related to men's coalitional behavior than to women's tending responses.

\section{References}

Arndt, J., Greenberg, J., \& Cook, A. (2002). Mortality salience and the spreading activation of worldviewrelevant constructs: Exploring the cognitive architecture of terror management. Journal of Experimental Psychology: General, 131, 307-324. doi:10.1037/0096-3445.131.3.307.

Arndt, J., Solomon, S., Kasser, T., \& Sheldon, K. M. (2004). The urge to splurge revisited: Further reflections on applying terror management theory to materialism and consumer behavior. Journal of Consumer Psychology, 14, 225-229. doi:10.1207/s15327663jcp1403_5.

Bercovitch, F. B., \& Strum, S. C. (1993). Dominance rank, resource availability, and reproductive maturation in female savanna baboons. Behavioral Ecology and Sociobiology, 33, 313-318. doi:10.1007/ BF00172929.

Bernhardt, P. C., Dabbs, J. R., Fielden, J. A., \& Lutter, C. D. (1998). Testosterone changes during vicarious experiences of winning and losing among fans at sporting events. Physiology \& Behavior, 65, 59-62. doi: 10.1016/S0031-9384(98)00147-4.

Bijleveld, E., Scheepers, D., \& Ellemers, N. (2012). The cortisol response to anticipated intergroup interactions predicts self-reported prejudice. Plos ONE, 7, doi: 10.1371/journal.pone.0033681.

Bingaman, E. W., Magnuson, D. J., Gray, T. S., \& Handa, R. J. (1994). Androgen inhibits the increases in hypothalamic corticotropin-releasing hormone (CRH) and $\mathrm{CRH}$-immunoreactivity following gonadectomy. Neuroendocrinology, 59, 223-234. doi:10.1159/000126663.

Bradley, K. I., \& Kennison, S. M. (2012). The effect of mortality salience on weapon bias. International Journal of Intercultural Relations, 36, 403-408. doi:10.1016/j.ijintrel.2011.09.006.

Burgess, L. H., \& Handa, R. J. (1992). Chronic estrogen-induced alterations in adrenocorticotropin and corticosterone secretion, and glucocorticoid receptor-mediated functions in female rats. Endocrinology, 13, 1261-1269. doi:10.1210/en.131.3.1261.

Byrd-Craven, J., Granger, D. A., \& Auer, B. J. (2011). Stress reactivity to co-rumination in young women's friendships: Cortisol, alpha-amylase, and negative affect focus. Journal of Social and Personal Relationships, 28, 469-487. doi:10.1177/0265407510382319.

Campbell, A. (1999). Staying alive: Evolution, culture, and women's intrasexual aggression. Behavioral and Brain Sciences, 22, 203-252. doi:10.1017/S0140525X99001818.

Critchlow, V., Liebelt, R. A., Bar-Sela, M., Mountcastle, W., \& Lipscomb, H. S. (1963). Sex difference in resting pituitary-adrenal function in the rat. American Journal of Physiology, 205, 807-815.

DeSoto, M.C., Hiltan, R.T., Deol, R-S.S., \& McAdams, D. (2010). Testosterone fluctuations in young men: The difference between interacting with like and not-like others. Evolutionary Psychology, 8, 173-188.

Fleming, A. S., Ruble, D., Krieger, H., \& Wong, P. Y. (1997a). Hormonal and experiential correlates of maternal responsiveness during pregnancy and the puerperium in human mothers. Hormones and Behavior, 31, 145-158. doi:10.1006/hbeh.1997.1376.

Fleming, A. S., Steiner, M., \& Corter, C. (1997b). Cortisol, hedonics, and maternal responsiveness in human mothers. Hormones and Behavior, 32, 85-98. doi:10.1006/hbeh.1997.1407.

Flinn, M. V. (2006). Evolution and ontogeny of stress response to social challenges in the human child. Developmental Review, 26, 138-174. doi:10.1016/j.dr.2006.02.003

Flinn, M. V., Ponzi, D., \& Muehlenbein, M. P. (2012). Hormonal mechanisms for regulation of aggression in human coalitions. Human Nature, 23, 68-88. doi:10.1007/s12110-012-9135-y.

Fuxjager, M. J., Mast, G., Becker, E. A., \& Marler, C. A. (2009). The 'home advantage' is necessary for a full winner effect and changes in post-encounter testosterone. Hormones and Behavior, 56, 214-219. doi:10. 1016/j.yhbeh.2009.04.009.

Gabrill, C., \& Kerns, K. A. (2000). Attachment style and intimacy in friendship. Personal Relationships, 7, 363-378.

Geary, D. C. (2010). Male, female: The evolution of human sex differences (2nd ed.). Washington, DC: American Psychological Association. doi:10.1037/12072-000.

Geary, D. C., \& Flinn, M. V. (2002). Sex differences in behavioral and hormonal response to social threat: Commentary on Taylor et al. (2000). Psychological Review, 109, 745-750. doi:10.1037/0033-295X.109.4.745.

Giardino, J., Gonzalez, A., Steiner, M., \& Fleming, A. S. (2008). Effects of motherhood on physiological and subjective responses to infant cries in teenage mothers: A comparison with non-mothers and adult. Hormones and Behavior, 53, 149-158. doi:10.1016/j.yhbeh.2007.09.010. 
Gleason, E. D., Fuxjager, M. J., Oyegbile, T. O., \& Marler, C. A. (2009). Testosterone release and social context: When it occurs and why. Frontiers in Neuroendocrinology, 30, 460-469. doi:10.1016/j.yfrne.2009.04.009.

Gonzalez-Bono, E. E., Salvador, A. A., Serrano, M. A., \& Ricarte, J. J. (1999). Testosterone, cortisol, and mood in a sports team competition. Hormones and Behavior, 35, 55-62. doi:10.1006/hbeh.1998.1496.

Gonzalez-Bono, E. E., Salvador, A., Ricarte, J. J., Serrano, M. A., \& Arnedo, M. M. (2000). Testosterone and attribution of successful competition. Aggressive Behavior, 26, 235-240. doi:10.1002/(SICI)10982337(2000)26:3<235::AID-AB3>3.0.CO;2-L.

Gordis, E. B., Granger, D. A., Susman, E. J., \& Trickett, P. K. (2006). Asymmetry between salivary cortisol and $\alpha$-amylase reactivity to stress: Relation to aggressive behavior in adolescents. Psychoneuroendocrinology, 31, 976-987. doi:10.1016/j.psyneuen.2006.05.010.

Granger, D. A., Kiviligan, K. T., Fortunato, C., Harmon, A. G., Hibel, L. C., Schwartz, E. B., \& Whembolua, G. L. (2007). Integration of salivary biomarkers into developmental and behaviorally-oriented research: Problems and solutions for collecting specimens. Physiology \& Behavior, 92, 583-590.

Greenberg, J., Pyszczynski, T., \& Solomon, S. (1986). The causes and consequences of a need for self-esteem: A terror management theory. In R. F. Baumeister (Ed.), Public self and private self (pp. 189-212). New York: Springer.

Greenspan, S. L., Rowe, J. W., Maitland, L. A., McAloon-Dyke, M., \& Elahi, D. (1993). The pituitary-adrenal glucocorticoid response is altered by gender and disease. Journal of Gerontology, 48, M72-M77. doi:10. 1093/geronj/48.3.M72.

Handa, R. J., Nunley, K. M., Lorens, S. A., Louie, J. P., McGivern, R. F., \& Bollnow, M. R. (1994). Androgen regulation of adrenocorticotropin and corticosterone secretion in the male rat following novelty and foot shock stressors. Physiology \& Behavior, 55, 117-124. doi:10.1016/0031-9384(94)90018-3.

Hrdy, S. B. (2007). Evolutionary context of human development: The cooperative breeding model. In C. A. Salmon \& T. K. Shackelford (Eds.), Family relationships: An evolutionary perspective (pp. 39-68). New York: Oxford University Press.

Kasser, T., \& Sheldon, K. M. (2000). Of wealth and death: Materialism, mortality salience, and consumption behavior. Psychological Science, 11, 348-351. doi:10.1111/1467-9280.00269.

Kivlighan, K. T., Granger, D. A., \& Booth, A. (2005). Gender differences in testosterone and cortisol response to competition. Psychoneuroendocrinology, 30, 58-71. doi:10.1016/j.psyneuen.2004.05.009.

McCormick, C. M., Linkroum, W., Sallinen, B. J., \& Miller, N. W. (2002). Peripheral and central sex steroids have differential effects on the HPA axis of male and female rats. Stress The International Journal on the Biology of Stress, 5, 235-247. doi:10.1080/1025389021000061165.

McGregor, H. A., Lieberman, J. D., Greenberg, J., Solomon, S., Arndt, J., Simon, L., \& Pyszczynski, T. (1998). Terror management and aggression: Evidence that mortality salience motivates aggression against worldview-threatening others. Journal of Personality and Social Psychology, 74, 590-605. doi:10.1037/ 0022-3514.74.3.590.

Nolan, S. A., \& Heinzen, T. E. (2008). Statistics for the behavioral sciences. New York: Worth.

Oxford, J., Ponzi, D., \& Geary, D. C. (2010). Hormonal responses differ when playing violent video games against an ingroup and outgroup. Evolution and Human Behavior, 31, 201-209. doi:10.1016/j. evolhumbehav.2009.07.002.

Pruessner, J., Kirschbaum, C., Meinlschmid, G., \& Hellhammer, D. H. (2003). Two formulas for computation of the area under the curve represent measures of total hormone concentration versus time-dependent change. Psychoneuroendocrinology, 28, 916-931.

Roelfsema, F., van den Berg, G., Frolich, M., Veldhuis, J. D., van Eljk, A., Buurman, M. M., \& Etman, B. H. (1993). Sex-dependent alteration in cortisol response to endogenous adrenocorticotropin. Journal of Clinical Endocrinology and Metabolism, 77, 234-240.

Routledge, C., Arndt, J., \& Goldenberg, J. L. (2004). A time to tan: Proximal and distal effects of mortality salience on sun exposure intentions. Personality and Social Psychology Bulletin, 30, 1347-1358. doi:10. $1177 / 0146167204264056$.

Rubin, K. H., Bukowski, W., \& Parker, J. G. (1998). Peer interactions, relationships, and groups. In W. Damon \& N. Eisenberg (Eds.), Handbook of child psychology:Vol. 3. Social, emotional, and personality development (pp. 619-700). New York: Wiley.

Rubinow, D. R., \& Schmidt, P. J. (1996). Androgens, brain, and behavior. The American Journal of Psychiatry, 153, 974-984.

Savin-Williams, R. C. (1979). Dominance hierarchies in groups of early adolescents. Child Development, 50, 923-935. doi:10.2307/1129316.

Solomon, S., Greenberg, J., \& Pyszczynski, T. (1991). A terror management theory of social behavior: The psychological functions of self-esteem and cultural worldviews. In M. P. Zanna (Ed.), Advances in experimental social psychology (Vol. 24, pp. 91-159). San Diego: Academic. 
Stallings, J., Fleming, A. S., Corter, C., Worthman, C., \& Steiner, M. (2001). The effects of infant cries and odors on sympathy, cortisol, and autonomic responses in new mothers and nonpostpartum women. Parenting: Science and Practice, 1, 71-100. doi:10.1207/S15327922PAR011\&2_5.

Stroud, L. R., Salovey, P., \& Epel, E. S. (2002). Sex differences in stress responses: Social rejection versus achievement stress. Biological Psychiatry, 52, 318-327. doi:10.1016/S0006-3223(02)01333-1.

Stroud, L. R., Foster, E., Papandonatos, G. D., Handwerger, K., Granger, D. A., Kivlighan, K. T., \& Niaura, R. (2009). Stress response and the adolescent transition: Performance versus peer rejection stressors. Development and Psychopathology, 21, 47-68. doi:10.1017/S0954579409000042.

Tabachnick, B. G., \& Fidell, L. S. (2007). Experimental design using ANOVA. Duxbury: England. Thompson Brooks/Cole.

Taylor, S. E. (2006). Tend and befriend: Biobehavioral bases of affiliation under stress. Current Directions in Psychological Science, 15, 273-277. doi:10.1111/j.1467-8721.2006.00451.x.

Taylor, S. E., Klein, L., Lewis, B. P., Gruenewald, T. L., Gurung, R. R., \& Updegraff, J. A. (2000). Biobehavioral responses to stress in females: Tend-and-befriend, not fight-or-flight. Psychological Review, 107, 411-429. doi:10.1037/0033-295X.107.3.411.

Uchino, B. N., Uno, D., \& Holt-Lunstad, J. (1999). Social support, physiological processes, and health. Current Directions in Pyschological Science, 8, 145-148.

Vaughn, J. E., Bradley, K. I., Byrd-Craven, J., \& Kennison, S. M. (2010). The effect of mortality salience on women's judgments of male faces. Evolutionary Psychology, 8, 477-491.

Wagner, J. D., Flinn, M. V., \& England, B. G. (2002). Hormonal response to competition among male coalitions. Evolution and Human Behavior, 23, 437-442. doi:10.1016/S1090-5138(02)00100-9.

Wisman, A., \& Koole, S. L. (2003). Hiding in the crowd: Can mortality salience promote affiliation with others who oppose one's worldviews? Journal of Personality and Social Psychology, 84, 511-526. doi: 10.1037/0022-3514.84.3.511. 